PALABRAS CLAVE

Calidad de la educación

Rendimiento escolar

Medición

Escuelas privadas

Escuelas públicas

Aspectos sociales

Argentina

María Marta Formichella

Docente Ayudante de las Cátedras

"Seminario de Contabilidad Económica"

y "Fundamentos de la Economía".

Departamento de Economía.

Universidad Nacional del Sur (UNS)

œmformichella@uns.edu.ar
REVISTA CEPAL 105 DICIEMBRE 2011

\section{¿Se debe el mayor rendimiento de las escuelas de gestión privada en la Argentina}

\section{al tipo de administración?}

\author{
María Marta Formichella
}

$\mathrm{E}$

objetivo del presente trabajo es analizar los factores determinantes de la calidad educativa en la Argentina y, especialmente, estudiar el papel del tipo de gestión escolar. Para cumplirlo, se utiliza un modelo de regresión multinivel y datos del Programa para la Evaluación Internacional de Alumnos (PISA, por sus siglas en inglés) del año 2006. Entre los principales resultados resalta que la correlación entre el tipo de gestión escolar (pública o privada) y el rendimiento educativo se disipa al considerar el entorno socioeconómico escolar. 


\section{I}

\section{Introducción}

En numerosos trabajos de investigación se ha planteado la importancia de la educación para el logro del desarrollo socioeconómico. La consideración de sus efectos benéficos va desde cuestiones vinculadas al mercado de trabajo hasta otras menos productivistas relacionadas con la mejor calidad de vida que pueden tener las personas educadas, en comparación con las que no lo están (Formichella, 2010).

En este contexto, resulta relevante el nivel de logros educativos al que puede acceder cada individuo. En la Argentina existen diferencias entre estos logros, tanto en cantidad como en calidad. Respecto de la cantidad, cabe mencionar que el $51 \%$ de las personas entre 25 y 65 años de edad no poseen título secundario y que el coeficiente de GINI educativo, calculado en función de los años de escolarización aprobados, asciende a 0,21. Por otra parte, en relación con la calidad, el índice de Theil - calculado con datos de las puntuaciones en ciencias de la prueba del año 2006 del Programa para la Evaluación Internacional de Alumnos (PISA) - es igual a 0,059 , en tanto que el $41 \%$ de la explicación de esta desigualdad se evidencia por diferencias entre las escuelas (Formichella, 2010).

De acuerdo con investigaciones previas en que se han estudiado los determinantes del rendimiento escolar para el caso argentino, puede decirse que las diferencias en los resultados de calidad educativa obedecen a cuestiones vinculadas a características de los individuos y sus hogares, y a factores relacionados con las particularidades de las escuelas; también en muchos trabajos aparece como relevante el entorno socioeconómico de los compañeros de clase.
Si bien tales investigaciones tienen numerosos puntos en común, hay una cuestión en la que discrepan y tiene que ver con el efecto del tipo de gestión escolar en los resultados educativos. Esta discrepancia se ha verificado también en estudios empíricos realizados en otros países ${ }^{1}$.

Como se verá, existen trabajos empíricos previos referidos a la educación argentina en los que se encuentra que la titulación de la escuela es muy importante en el rendimiento de sus alumnos, mientras que en otros se halla que el efecto de esta se disipa al considerar el entorno socioeconómico de quienes asisten a cada tipo de escuela.

Por ello, el objetivo de este trabajo es ensayar una respuesta en torno de la mencionada diferencia. La hipótesis que se plantea opera en favor de la segunda postura presentada, es decir, que la influencia de la gestión en el desempeño de los alumnos oculta en realidad el efecto que el entorno socioeconómico escolar tiene en el rendimiento.

Para cumplir con el objetivo propuesto y luego de esta Introducción se presentan las secciones siguientes. En la sección II se describen los principales trabajos previos cuyo objetivo ha sido estudiar los determinantes del rendimiento escolar en la Argentina; en la sección III se da cuenta de la fuente de datos aquí usada; en la sección IV se explica la metodología utilizada, y en la sección V se detallan los modelos propuestos y los resultados derivados de ellos. Por último, en la sección VI se presentan las conclusiones del trabajo.

\footnotetext{
${ }^{1}$ Véase una revisión de estos trabajos en Calero y Escardíbul (2007).
}

\section{II}

\section{Antecedentes sobre el tema en la Argentina}

Existen diversos trabajos empíricos con respecto a la Argentina en que se estudian los determinantes del rendimiento escolar. Cervini (1999) analiza el desempeño del rendimiento educativo (medido como los resultados en la evaluación de matemáticas) de los alumnos de séptimo año de educación general básica (EGB), para lo cual utiliza información del Operativo Nacional de Evaluación (ONE) de la calidad educativa del año 1997. La metodología que aplica es un análisis jerárquico de dos niveles: escuelas y alumnos. El autor encuentra que en el rendimiento existe un importante efecto del nivel socioeconómico familiar y del entorno escolar. 
Además, constata que los siguientes factores afectan negativamente a los resultados: i) la edad (que representa el problema de la extraedad ${ }^{2}$ ); ii) haber repetido previamente; iii) haber cambiado de escuela; iv) vivir lejos del establecimiento, y v) ser mujer. Por otra parte, encuentra que inciden positivamente variables vinculadas a la automotivación, el esfuerzo personal y la percepción del alumno respecto de la ayuda que recibe de su familia. Finalmente, halla evidencia de que una mejor infraestructura escolar y recursos educativos de mayor calidad producen efectos positivos en el rendimiento y, al mismo tiempo, considera que el tipo de gestión del colegio no resulta ser una variable explicativa relevante.

Este mismo autor, en el año 2002, estudia nuevamente los determinantes del rendimiento escolar en el nivel primario, utilizando también datos del oNE de 1997. Para ello, usa como metodología el análisis jerárquico considerando ahora tres niveles: provincias, escuelas y alumnos. De este modo, encuentra que la explicación más gravitante de las diferencias en resultados la proporciona el nivel de alumnos y luego el de escuelas, y que el nivel de provincias es prácticamente irrelevante. $\mathrm{Su}$ principal conclusión es que en la Argentina se cumple la teoría de la reproducción cultural, ya que el rendimiento se explica en gran medida por el capital cultural y económico del hogar. También detecta que el efecto de los factores socioeconómicos varía significativamente entre escuelas, por lo que deduce que estas difieren en su capacidad de compensar las desigualdades de origen. Por último, concluye que - dentro del conjunto de factores vinculados a la escuela- el que más incide en el rendimiento es el contexto socioeconómico de los compañeros de clase.

Asimismo, Cervini (2002a) analiza los determinantes del rendimiento educativo en el nivel medio, utilizando para ello datos del Censo Nacional de Finalización del Nivel Secundario del año 1998. Nuevamente emplea la metodología de análisis jerárquico considerando tres niveles: provincias, escuelas y alumnos. Al igual que en el caso anterior, obtiene que la desigualdad en resultados se evidencia mayormente entre individuos, pero que también un elevado porcentaje se explica por diferencias entre escuelas, mientras que el nivel de provincias no aporta demasiado a la mencionada disparidad. Además, constata que existe una correlación negativa entre el género femenino (masculino) y el rendimiento

\footnotetext{
${ }^{2}$ La extraedad se refiere a los alumnos con dos o más años de retraso escolar, y expresa en qué medida los estudiantes de distintos grupos etarios están asistiendo a cursos inferiores a los esperados en función de su edad.
}

en matemática (lengua), que el capital económico del hogar es determinante en el acceso al nivel medio y que el capital cultural del hogar es el que afecta positivamente al desempeño. De nuevo, halla que las escuelas difieren en su capacidad de compensar las desigualdades de origen.

Por su parte, Fernández Aguerre (2002) utiliza datos del oNE del año 2000 y una metodología de análisis de regresión logística para estudiar los determinantes del éxito escolar en los alumnos de sexto año en la Argentina. Al igual que Cervini, encuentra evidencia en favor de una correlación positiva entre el nivel socioeconómico del hogar del alumno y su rendimiento. Además, observa que la composición sociocultural de la escuela tiene más peso en el resultado que el capital económico del hogar. Por último, cabe señalar que constata que los alumnos que asisten a escuelas privadas tienen un mayor rendimiento que los que asisten a escuelas públicas; y que los varones presentan un mejor rendimiento que las mujeres.

Wößmann y Fuchs (2005) también estudian el caso argentino utilizando regresiones lineales robustas por clusters (metodología CRLR, por sus siglas en inglés: clustering-robust linear regressions) y tomando datos del Estudio Internacional del Progreso en Competencia Lectora (PIRLS, por sus siglas en inglés) de 2001, efectuado en el nivel educativo primario. Estos autores constatan que los factores vinculados a las características y antecedentes familiares del alumno son los principales a la hora de explicar el rendimiento educativo. Además, no encuentran una evidencia manifiesta sobre la relación entre el desempeño educacional y la dotación de recursos de la escuela u otras características escolares. Solo hallan que asistir a una escuela que se rija por un currículum centralizado o cuyas clases de formación se basen en habilidades afecta positivamente a ese desempeño.

Luego, Cervini (2005a y 2006) profundiza su trabajo del año 2002 dedicado al nivel medio centrándose en el área de matemáticas. Encuentra que ser mujer, repitente o trabajar una mayor cantidad de horas disminuye el rendimiento escolar y que existe un alto grado de selectividad institucional de acuerdo con las características socioeconómicas de los alumnos. Además, halla evidencia en favor de una correlación positiva entre el entorno escolar y el rendimiento. Verifica también que las escuelas difieren en su capacidad de compensar las diferencias provenientes del hogar y que la inequidad referida a la desigualdad del efecto de las variables entre escuelas disminuye cuando se analizan escuelas de similar composición.

Asimismo, Cervini (2005b) estudia los resultados educativos, pero no solo los cognitivos, sino también los no cognitivos referidos a actitudes socioeducativas 
generales (medidos mediante otras variables que surgen del cuestionario de los alumnos y con las que se intenta captar cuestiones subjetivas vinculadas a estos). Para ello utiliza un modelo jerárquico de tres niveles: el estudiante, la escuela y la provincia, y toma datos del Censo Nacional de Finalización del Nivel Secundario del año 1998. Al comparar los determinantes de ambos tipos de resultados encuentra sobre todo lo siguiente: i) las características individuales afectan más a los resultados cognitivos que a los no cognitivos; ii) las escuelas difieren en su capacidad para compensar las desigualdades de origen en relación con los resultados de ambos tipos; y iii) las escuelas son poco consistentes en la obtención de resultados de sus alumnos (no hay altas correlaciones entre los resultados cognitivos y los no cognitivos).

Por su parte, Gertel y otros (2006) también utilizan datos del ONE para estudiar los determinantes del rendimiento educativo en la Argentina. Lo hacen con los resultados de las pruebas de matemática y lengua de nivel primario del año 2000. Aplican como metodología un modelo jerárquico de dos niveles: estudiantes y aula. Entre sus resultados se destaca el efecto de las variables repitencia y género: ser repitente disminuye el rendimiento y ser varón lo aumenta (disminuye) en matemática (lengua). Con respecto a las variables del hogar, sobresale que poseer hermanos que hayan abandonado el colegio o nunca hayan asistido a este aminora el rendimiento y que el nivel socioeconómico se relaciona positivamente con los resultados educativos, aunque su magnitud es pequeña. Por último, encuentran que tienen una repercusión relevante ciertas variables vinculadas al establecimiento escolar, entre ellas: calidad edilicia, experiencia y capacitación del docente y tipo de gestión de la escuela (los alumnos que asisten a colegios de gestión privada obtienen mejores resultados).

Posteriormente, dada la importancia de los datos que encontraron respecto de la gestión escolar, Gertel y otros (2007a) plantean un modelo de tres niveles: alumnos, cursos y escuelas, donde el tercer nivel (en este caso las escuelas) está representado sobre todo por variables vinculadas a la gestión. En sus resultados ratifican la idea de la relevancia de la titulación por sobre los resultados educativos.

Estos mismos autores (Gertel y otros, 2007b) analizan nuevamente el rendimiento educativo, pero considerando no solo datos del ONE, sino también de la prueba internacional Laboratorio Latinoamericano de Evaluación de la Calidad de la Educación (LLECE), que fue realizada en 1997 con alumnos de nivel educativo primario. Utilizan el análisis de datos jerárquico y los mismos tres niveles que en el caso anterior. Entre los principales resultados muestran que la gestión es un factor muy relevante a la hora de explicar el desempeño en los resultados educativos en la Argentina.

Por otra parte, Santos (2007) estudia los determinantes de la calidad educativa en la Argentina, pero respecto del nivel medio. Para ello utiliza datos de la prueba PISA del año 2000, toma en cuenta los resultados de las evaluaciones de las competencias en las áreas de lengua y matemática y aplica la metodología de análisis de regresión por quantiles. Entre sus principales resultados encuentra que el género es una variable determinante, ya que observa que las mujeres presentan mejor resultado en lengua y los varones en matemática. Asimismo, constata que el tamaño de la clase no debiera superar los 32 alumnos para un resultado de aprendizaje positivo y que la calidad de los recursos educativos de la escuela tiene un efecto importante en el desempeño. También detecta que luego de controlar por medio de otras características, las escuelas privadas no muestran un resultado superior al de las públicas, y plantea que esto puede deberse a que las primeras albergan a los alumnos de mejor estrato socioeconómico. Por último, cabe señalar que halla muy significativo el hecho de que los estudiantes dispongan de recursos educativos en sus hogares.

En igual sentido, Abdul-Hamid (2007) analiza también los determinantes de la calidad educativa en la Argentina usando regresiones por quantiles y datos de la prueba PISA del año 2000. De este modo, observa que afectan positivamente al rendimiento educativo -entre otros factores - los siguientes aspectos: i) el clima de aprendizaje escolar; ii) la localización geográfica de la escuela (las localizadas en ciudades más grandes presentan mejor desempeño); iii) el hecho de que los alumnos sean orientados y alentados en su aprendizaje; iv) el tipo de gestión escolar (los alumnos de las escuelas privadas tienen mayor rendimiento); v) que las mujeres sean mayoría en la escuela; vi) que el alumno cuente con recursos educativos en el hogar, y vii) que el nivel educativo de la madre del alumno supere el nivel medio.

Por último, cabe mencionar dos trabajos realizados por Cervini en que compara los niveles educativos primario y medio en la Argentina utilizando datos del ONE (2000) y del Censo Nacional de Finalización del Nivel Secundario (1998), así como una metodología de análisis multinivel.

Por una parte, en el primer trabajo (Cervini, 2009) el autor utiliza tres niveles: alumnos (nivel 1), escuelas (nivel 2) y provincias (nivel 3), con el fin de comparar la estructura de (in)equidad en la distribución de los 
rendimientos en las evaluaciones de las asignaturas de lengua y matemática. Entre otros resultados, detecta que las escuelas difieren ampliamente en el desempeño de sus alumnos en las evaluaciones en los dos niveles educativos considerados y que las diferencias en su interior son mayores en la educación primaria; de ello deduce que existe un efecto más acentuado de las características familiares en ese tramo educacional. Asimismo, halla que - en general - todas las variables del entorno socioeconómico del alumno afectan a los resultados de las pruebas en los dos niveles educativos, y que las variables de contexto escolar también son determinantes de peso en ambos, aunque observa que en la eduación primaria es más relevante el entorno económico de los compañeros y en la secundaria gravita más el clima educativo representado por la educación de los padres de los alumnos.

Por otra parte, en el segundo trabajo (Cervini, 2010) este autor emplea varios modelos en que desagrega la información en diferentes niveles (considera, alternativamente, tres de los siguientes: alumno, aula, escuela, municipio y provincia) y se focaliza en el análisis y comparación del "efecto escuela" entre los

\section{III}

\section{Los datos}

El análisis de regresión propuesto en este trabajo ${ }^{3}$ se realiza con datos provenientes del programa PISA, año 2006, implementado por la Organización de Cooperación y Desarrollo Económicos (OCDE), cuyo objetivo es medir las competencias de los estudiantes de 15 años de diferentes países a fin de evaluar cuán preparados están para enfrentar los desafíos que tendrán en su vida adulta viviendo en sociedad. El estudio consta de una serie de evaluaciones de las capacidades de los estudiantes en matemática, ciencias y lengua. Este programa se realiza trianualmente y en cada oportunidad se elige un área de evaluación como prioritaria. En la prueba PISA del año 2006 se hizo hincapié en la competencia científica (OCDE, 2006a).

Las evaluaciones del programa PISA se complementan con información sobre las características del hogar de cada individuo y respecto de las condiciones

\footnotetext{
${ }^{3}$ La metodología de regresión econométrica se explica en la sección IV.
}

niveles educativos primario y medio. Concluye que los resultados están sujetos a la metodología utilizada, que el efecto neto de la escuela en la Argentina se acerca al de los países desarrollados y que no hay diferencias significativas entre los niveles educativos primario y secundario.

Para finalizar esta sección y a modo de síntesis, cabe mencionar que entre los trabajos aludidos parece haber consenso en cuanto al efecto del estrato socioeconómico del hogar en los resultados educativos, y también con respecto al "entorno escolar". Además, en relación con la variable que más interesa, de acuerdo con el objetivo aquí planteado, se observa que algunos autores utilizan la titulación de la escuela como variable explicativa, mientras que otros la omiten. Asimismo, entre quienes la emplean no existe consenso; algunos concluyen que es una variable importante a la hora de explicar el rendimiento escolar (Fernández Aguerre, 2002; Gertel y otros, 2007a y 2007b; Abdul-Hamid, 2007), mientras que otros consideran que pierde influencia e incluso es irrelevante cuando se controla el modelo por variables del entorno socioeconómico escolar (Cervini, 2010; Santos, 2007). de la escuela a que asiste. Los datos acerca del hogar se relevan por medio de un cuestionario que completan los alumnos en el momento de realizar la evaluación, y en algunos países se complementa con un cuestionario que responden los padres. La información referida a las escuelas surge de las entrevistas a sus directores.

Las puntuaciones de la prueba PISA se encuadran en un rango que va de 0 a 1.000 y se distribuyen en seis estratos. Los valores extremos de cada estrato varían según el área de competencia bajo análisis (OCDE, 2006a).

Los resultados de las evaluaciones se presentan utilizando "valores plausibles" (PV) que son una representación del conjunto de capacidades en un estudiante. Generar valores plausibles a partir de una evaluación significa obtener un continuo sobre la base de un grupo de variables discontinuas (las puntuaciones de la prueba) (OCDE, 2003).

Martínez Arias (2006) explica que el problema a resolver en el programa PISA es que cada individuo responde solo a cierto número de ítems y se vuelve 
necesario estimar cómo hubiera contestado en la totalidad de los casos. Esas respuestas se predicen en función de las preguntas que sí se han contestado y de otras variables denominadas de "condicionamiento" surgidas del cuestionario de contexto. Así, en lugar de hacerse una única predicción se genera una distribución de valores a posteriori para cada individuo, cada uno con sus probabilidades asociadas, y de esa distribución se obtienen aleatoriamente cinco valores, que son los ya mencionados "valores plausibles". De esta manera, se evita el sesgo que existiría si se predijera la habilidad (no observable) en función de un número limitado de datos observables. Para estimar estos valores, el equipo del programa PISA utiliza un software específico.

Asimismo, es menester mencionar que los estadísticos poblacionales y los parámetros de los modelos de regresión deben estimarse usando los valores plausibles separadamente. El valor de cada estadístico poblacional que se desee calcular será igual al promedio que surge de estimar dicho estadístico poblacional con cada uno de los cinco valores plausibles que informa la prueba PISA. Lo mismo sucede en el caso de los parámetros de los modelos, en cuyo caso el valor de cada parámetro será igual al promedio que surge de estimar dicho parámetro con cada uno de los cinco valores plausibles (OCDE, 2003).

Si bien los países que participan en el programa PISA no son siempre exactamente los mismos, ellos se dividen en forma constante en dos grupos: los pertenecientes a la OCDE y los asociados a la OCDE. La Argentina forma parte del segundo conjunto y participó en las pruebas PISA de los años 2000, 2006 y 2009.

\section{IV}

\section{Metodología}

Para cumplir con el objetivo de este trabajo se aplican técnicas de regresión multinivel. Cervini (1999) plantea que esta es una técnica de correlación adecuada para estudiar las variaciones en las características de los individuos que forman parte de grupos (por ejemplo, en este caso, escuelas).

En los análisis de regresión multinivel se tiene en cuenta que las unidades muestrales están anidadas dentro de unidades más amplias. En lugar de calcular una ecuación de regresión sobre el conjunto de datos entero, se calcula una ecuación de regresión por cada
En el momento de realizar este trabajo los últimos datos disponibles son los de PISA 2006 y por ello es la base de datos elegida. En la Argentina, la prueba PISA 2006 se aplicó entre el 14 y el 16 de agosto de ese año. Fueron evaluados 4.339 alumnos de 15 años pertenecientes a 179 escuelas diferentes. En el presente trabajo, la muestra se redujo a 3.860 observaciones debido a la existencia de datos perdidos. Sin embargo, según la OCDE (2003) esto no causa distorsiones en los resultados porque la merma de datos fue cercana al $10 \%^{4}$.

Por último, cabe señalar que en el programa PISA la muestra de alumnos evaluados en cada país se realiza mediante un procedimiento que comprende dos etapas: primero se seleccionan las escuelas y luego los alumnos dentro de estas. Por ello, como se verá en la próxima sección, es sobre todo recomendable utilizar modelos multinivel para estudiar el rendimiento escolar.

\footnotetext{
${ }^{4}$ Para no perder más observaciones, algunas variables no se utilizan en el análisis. A fin de recuperar esas variables no se aplican técnicas de imputación complejas, porque estas implican estimar el valor de la variable faltante mediante regresiones en que se utilice el resto de las variables explicativas, y para varias observaciones faltaba el dato de más de una variable. Santos (2007) explica que esto puede provocar problemas más que brindar soluciones. En dichos casos tampoco se aplica la técnica de imputación sencilla (que consiste en reemplazar el dato perdido por el valor medio de la muestra), porque al ser muy alto el porcentaje de datos faltantes, no utilizar una forma de imputación fundamentada en las características de la observación puede distorsionar los resultados. Sin embargo, como se verá más adelante, en algunos casos (variables CULTPOSS, SCMATEDU y CLSIZE) sí se aplica la técnica de imputación sencilla debido a que el porcentaje de datos perdidos es bajo.
}

una de dichas unidades más amplias (OCDE, 2003). Por ello, cuando los datos se encuentran agrupados estos modelos son más apropiados, ya que incorporan esta información respecto del anidamiento.

Además, cuando la muestra de la población es seleccionada por etapas se está en presencia de un caso de análisis multinivel jerárquico y no existe independencia de las observaciones dentro de cada grupo (Hox, 1995). Es decir, cuando primero se selecciona una estructura mayor y luego, dentro de la misma, se seleccionan casos, existe una estructura multinivel. Una 
ventaja de utilizar el análisis multinivel en este caso es que posibilita estudiar, al mismo tiempo, los efectos de las variables grupales e individuales en los resultados individuales y, además, incorpora la consideración de la dependencia de las observaciones dentro de cada grupo (Diez Roux, 2002).

De este modo, el uso de modelos multinivel permite obtener mejores estimadores de los coeficientes de regresión y de su variación, en comparación con los modelos tradicionales representados principalmente por los modelos de mínimos cuadrados ordinarios (De la Cruz, 2008). Además, los tests estadísticos estándar se apoyan fuertemente en el supuesto de independencia de las observaciones y si este es violado (como ocurre en las estructuras multinivel), las estimaciones de los errores estándar de los tests estadísticos convencionales son mucho más pequeñas y sus resultados "significativos" espurios (Hox, 1995).

Un ejemplo de estructura multinivel es el caso de las escuelas que agrupan clases, las que a su vez agrupan alumnos. Por ello, este tipo de análisis resulta conveniente cuando se estudian los determinantes del rendimiento educativo (Hox, 1995; Bryk y Raudenbusch, 1988, citado en Calero, Choi y Waisgrais, 2009). Cuando se tienen en cuenta los tres niveles mencionados se plantea un modelo jerárquico de tres niveles, y cuando solo se dispone de información sobre las escuelas y los alumnos se plantea un modelo jerárquico de dos niveles.

Por último, cabe señalar que los modelos multinivel pueden incorporar efectos fijos y aleatorios. La forma más simple consiste en que la variabilidad entre los grupos (efectos aleatorios) se evidencie solo en el intercepto, y formas más complejas aparecen cuando esta variabilidad se incorpora en los coeficientes de las variables explicativas. En el primer caso, existe una recta de regresión para cada grupo y todas las líneas son paralelas; mientras que en el segundo caso las rectas también poseen pendientes diferentes. Cabe destacar que el hecho de que el intercepto se defina con efectos aleatorios es básico; de lo contrario, si se definiera con efectos fijos, no tendría sentido aplicar un modelo de análisis de multinivel (OCDE, 2003).

Dado que los modelos jerárquicos presentan diferentes categorías, existen variables en cada una de ellas. Por ejemplo, si se considera el caso del rendimiento escolar en el que existen dos niveles: escuelas ${ }^{5}$ y alumnos, habrá algunas variables que caracterizarán a las escuelas (nivel 2) y otras a los alumnos (nivel 1).

\footnotetext{
${ }^{5}$ En el programa PISA se usa la palabra centro como sinónimo de escuela.
}

Las variables del nivel 2 serán idénticas para todos los alumnos dentro de una escuela determinada; por ello, estas variables solo influirán en los interceptos de las escuelas. Las variables del nivel 1 podrán ser incorporadas con efectos fijos o aleatorios en función de la teoría referida al tema de estudio y de los objetivos de investigación (OCDE, 2003). Si una variable de nivel 1 se incorpora con efectos fijos, se está suponiendo que no hay diferencia entre las escuelas respecto del efecto de dicha variable en la variable dependiente. En cambio, si una variable de nivel 1 se incluye asignándole efectos aleatorios, se está considerando que su efecto en la variable dependiente difiere entre escuelas.

En el caso del rendimiento escolar, si los efectos son fijos los coeficientes de regresión brindan información sobre qué ocurre con el resultado educativo - dentro de un centro- cuando se modifica el valor de una variable explicativa (ceteris paribus). Dado que los efectos son fijos, dicho coeficiente es igual para todos los centros (OCDE, 2003).

Asimismo, si los efectos son aleatorios los coeficientes se interpretan de igual modo, pero poseen un valor diferente dentro de cada centro. Dichos coeficientes pueden dividirse en dos partes, una fija, compartida por todas las escuelas, y una aleatoria, que representa la distancia residual entre el coeficiente de cada centro y la parte común (OCDE, 2003).

Formalmente, suponiendo que hay un modelo de rendimiento educativo conformado por dos niveles y que incorpora tres variables explicativas, una en el nivel 2 (P) y dos en el nivel 1 ( $\mathrm{X}$ de efectos fijos y $\mathrm{Z}$ de efectos aleatorios), lo explicado en los párrafos anteriores puede expresarse de la siguiente manera:

- Nivel 1:

$$
Y_{i j}=\beta_{0 j}+\beta_{1 j} X_{i j}+\beta_{2 j} Z_{i j}+r_{i j}
$$

- Nivel 2:

$$
\begin{gathered}
\beta_{0 j}=\gamma_{00}+\gamma_{01} P_{j}+\mu_{0 j} \\
\beta_{1 j}=\gamma_{10} \\
\beta_{2 j}=\gamma_{20}+\mu_{2 j}
\end{gathered}
$$

- Modelo completo:

$$
Y_{i j}=\gamma_{00}+\gamma_{01} P_{j}+\gamma_{10} X_{i j}+\gamma_{20} Z_{i j}+\mu_{0 j}+\mu_{2 j} Z_{i j}+\mathrm{r}_{i j}
$$

donde:

$Y_{i j} \quad$ es el resultado educativo (esperado) del alumno " $i$ " en la escuela " $j "$ ". 
$\beta_{0 j}$ es el intercepto de la recta de regresión para la escuela " $j$ ".

$\beta_{1 j}$ es el coeficiente que acompaña a la variable explicativa $X$, que está incorporada en el modelo con efectos fijos; por lo tanto, $\beta_{1 j}$ es igual para todos los centros y se representa mediante $\gamma_{10}$.

$\beta_{2 j}$ es el coeficiente que acompaña a la variable explicativa $Z$, que está incorporada en el modelo con efectos aleatorios; por lo tanto, varía entre centros. Está formada por una parte fija $\left(\gamma_{20}\right)$ y una parte aleatoria $\left(\mu_{2 j}\right)$. Esta última representa la distancia residual desde el coeficiente de regresión de los centros hasta $\gamma_{20}$

$\gamma_{01}$ es el coeficiente que acompaña a la variable explicativa $P$ (primera y única del nivel 2 , por eso el subíndice es "01"). Dado que pertenece al nivel 2 , existe un valor de $P$ por cada centro " "j". Como puede observarse en la ecuación, esta variable influye en el valor del intercepto $\beta_{0 j}$.

$r_{i j} \quad$ es la varianza residual dentro de cada centro.

$\mu_{0 j}$ es la varianza residual entre centros.

En los análisis multinivel resulta útil estimar un modelo sin incluir variables explicativas, al que se denomina "modelo nulo"; este brinda información sobre qué proporción de la desigualdad en los resultados de rendimiento educativo se debe a diferencias entre centros y qué proporción se vincula a desigualdades en su interior. Formalmente, el modelo se expresa así:

donde:

$$
Y_{i j}=\gamma_{00}+\mu_{0 j}+r_{i j}
$$

$\gamma_{00}$ representa los efectos fijos o determinísticos (intercepto global).

$\mu_{0 j}$ y $r_{i j}$ representan los efectos aleatorios o estocásticos. En este caso, los interceptos de cada centro ( $\operatorname{los} \beta_{0 j}$ ) son iguales a las medias de los centros o muy cercanos a estas. Como puede observarse, $\beta_{0 j}$ está formado por una parte fija - común a todas las escuelas $\left(\gamma_{00}\right)$ - y una parte aleatoria $\left(\mu_{0 j}\right)$, que representa la desviación de la escuela " $j$ " respecto de $\gamma_{00}$. Cabe señalar que $\gamma_{00}$ es el resultado del conjunto de escuelas (promedio) y es denominado "intercepto global". Entonces, $\mu_{0 j}$ es la desviación de la escuela " $j$ " del promedio del conjunto de las escuelas y representa la varianza entre ellas.

Por otra parte, $r_{i j}$ es el desvío del resultado del individuo " $i$ " respecto del promedio de la escuela " $j$ " a que pertenece. Puesto que en este modelo se le asigna a cada alumno el promedio de su escuela como puntuación predicha, $r_{i j}$ es igual a la varianza dentro de cada escuela.
Entonces, cuando no hay variables explicativas en el modelo, las varianzas residuales intracentros e intercentros son iguales a las estimaciones de las varianzas intracentros e intercentros (OCDE, 2003).

En síntesis, habitualmente existen dos tipos de índices relevantes en los análisis multinivel: los coeficientes de regresión y la descomposición de la varianza entre los distintos niveles. Del segundo índice se deriva un indicador muy utilizado denominado "coeficiente de correlación intraclase" $(\rho)$, que representa la proporción de la varianza residual explicada por diferencias entre escuelas $\left(\rho=\mu_{0 j} /\left(\mu_{0 j+} r_{i j}\right)\right.$. En el caso del modelo nulo, este representa la proporción de la varianza de los resultados educativos entre escuelas. Si ese valor fuera cero (0) no tendría sentido plantear un modelo multinivel.

En los modelos que incluyen variables explicativas, los análisis multinivel suelen informar sobre la varianza del residuo, es decir, aquella varianza de los resultados que no se explica mediante las variables predictoras incorporadas en el modelo. Sin embargo, es interesante conocer la varianza explicada, que puede obtenerse al comparar el modelo propuesto con el modelo nulo para cada nivel y de forma global. La manera de calcular la varianza se explica en el cuadro 1.

Asimismo, cabe describir la forma general de los modelos econométricos que se utilizan en este trabajo. Como se detalló en la sección anterior, no se cuenta con datos a nivel de las clases, por lo que se utilizan modelos jerárquicos de dos niveles: alumnos y escuelas. A la vez, se permite la variación entre escuelas del término constante y de las pendientes, es decir, que no solo se permite la variabilidad en los interceptos de las rectas de cada escuela, sino también en los coeficientes que acompañan a algunas de las variables explicativas del nivel 1. A continuación se presenta la expresión formal de los modelos:

CUADRO 1

\section{Porcentaje de varianza explicado por las} variables sobre el modelo nulo \begin{tabular}{ll}
\hline Total & $1-\left(\mu_{0 j}+r_{i j}\right)$ Modelo propuesto/ $\left(\mu_{0 j}+r_{i j}\right)$ Modelo nulo \\
$\begin{array}{l}\text { Nivel 1 } \\
\text { (alumnos) }\end{array}$ & $1-\left(r_{i j}\right)$ Modelo propuesto/ $\left(r_{i j}\right)$ Modelo nulo \\
$\begin{array}{l}\text { Nivel 2 } \\
\text { (escuelas) }\end{array}$ & $1-\left(\mu_{0 j}\right)$ Modelo propuesto $/\left(\mu_{0 j}\right)$ Modelo nulo
\end{tabular}

Fuente: elaboración propia sobre la base de Organización de Cooperación y Desarrollo Económicos (OCDE), El programa PISA de la OCDE. ¿Qué es y para qué sirve?, París, 2003. 
Nivel 1

$$
Y_{i j}=\beta_{0 j}+\sum_{k=1}^{K} \gamma_{k 0} X_{k i j}+\sum_{m=1}^{M} \beta_{\mathrm{mj}} Z_{m i j}+r_{i j}
$$

Nivel 2

$$
\begin{gathered}
\beta_{0 j}=\gamma_{00}+\sum_{f=1}^{F} \gamma_{0 f} P_{j}+u_{0 j} \\
\beta_{m j}=\gamma_{m 0}+u_{m j}
\end{gathered}
$$

donde: $K$ es el número de variables explicativas de nivel 1 que se incorporan al modelo con efectos fijos $(X) ; M$ es el número de variables explicativas de nivel 1 que se incorporan al modelo con efectos aleatorios $(Z)$, y $F$ el número de variables explicativas de nivel 2 $(P)$. El resto de la nomenclatura ya ha sido explicada previamente.

En este punto, cabe mencionar que en el trabajo se utiliza el programa computacional "modelo jerárquico lineal" (WHLM, por sus siglas en inglés) para calcular los parámetros de las ecuaciones anteriores, los que se estiman simultáneamente mediante métodos iterativos que maximizan una función de máxima verosimilitud.

En todos los modelos que aquí se presentan se ponderan las observaciones con la variable-peso $w_{-} f_{s t u w t}{ }^{6}$ proporcionada en la base de datos del programa PISA y se normalizan los pesos con la opción específica del programa computacional WHLM elegido para trabajar. Además, se utilizan como variable dependiente los cinco valores plausibles de la variable "puntuación en ciencias" (por tratarse del área prioritaria en PISA 2006) y el programa brinda el resultado promedio final de los estimadores poblacionales. Por último, con el fin de evitar posibles problemas en los tests de significatividad en caso de existir heteroscedasticidad, se calculan los parámetros de las ecuaciones y sus respectivos errores estándar robustos a la heteroscedasticidad.

6 "Los pesos son inversamente proporcionales a la probabilidad de selección" (OCDE, 2003).

\section{Los modelos planteados y los resultados encontrados}

A fin de cumplir con el objetivo propuesto, en esta sección se plantea emplear modelos multinivel. Como ya se ha mencionado, para que sea adecuado y tenga sentido aplicar un modelo de análisis jerárquico, es necesario que existan diferencias entre las unidades de anidamiento, en este caso las escuelas. Por ello, a objeto de corroborar que es recomendable utilizar modelos multinivel, se analiza cuánto de la divergencia de rendimiento se debe a disparidades entre las escuelas y cuánto a diferencias en su interior. Para eso se estima el modelo nulo tal como se ha explicado en la sección IV (metodología), y se lo denomina modelo 1.

Como puede observarse en el cuadro 2 , más de la mitad de las diferencias en rendimiento obedece a disparidades entre las escuelas, lo que denota que existen considerables desigualdades entre ellas. Entonces, se corrobora la conveniencia de utilizar modelos multinivel para estudiar los determinantes del rendimiento escolar. Cervini (2002b) explica que esto es importante a fin de poder separar la variabilidad de cada grupo y mejorar la calidad de las estimaciones.

Dentro del análisis de los posibles factores vinculados al rendimiento educativo, aquí interesa especialmente estudiar el efecto del tipo de gestión escolar. Se observa que, en promedio, las escuelas de gestión privada obtienen una mayor puntuación en ciencias que las escuelas de gestión pública (442,34 versus 377,02$)^{7}$. Se trata entonces de analizar si dichas diferencias son significativas en relación con el tipo de gestión, o si son otros los factores vinculados a esa disparidad. Para ello, en función de trabajos previos (Calero y Escardíbul, 2007; Santos, 2007; Cervini, 2002a, 2005a, 2005b y 2009; entre otros), parece relevante incluir y analizar

\footnotetext{
${ }^{7}$ El porcentaje de escuelas privadas independientes - es decir, no subsidiadas - es muy pequeño $(7,9 \%)$, por lo que se las agrupa con las escuelas de gestión privada subsidiadas.
} 
CUADRO 2

\section{Resultados del modelo nulo}

\begin{tabular}{lc}
\hline Variable dependiente & $\begin{array}{c}\text { Valores plausibles } \\
\text { en ciencias }\end{array}$ \\
\hline Valor del intercepto & 366,51 \\
Varianza en el interior de los centros $\left(\sigma^{2}\right)$ & 4143,37 \\
Varianza entre centros $\left(\tau^{2}\right)$ & 5725,91 \\
Varianza total & 9869,28 \\
Porcentaje de varianza residual existente por & $42 \%$ \\
diferencias entre alumnos & $58 \%$ \\
$\begin{array}{l}\text { Porcentaje de varianza residual existente por } \\
\text { diferencias entre centros }(\rho)\end{array}$ & \\
\hline
\end{tabular}

Fuente: elaboración propia sobre la base de datos del Programa para la Evaluación Internacional de Alumnos (PISA), 2006.

el contexto escolar al que se enfrentan los individuos, también denominado "efecto de pares" (peers efects).

El efecto de pares se fundamenta en que el individuo no aprende únicamente del profesor, sino que interactúa con sus compañeros y de esa interrelación surgen aprendizajes tanto en competencias como en conocimientos. Asimismo, se transmiten una serie de estímulos, tales como motivación y pautas de comportamiento, que muchas veces reflejan indirectamente hábitos de los padres (Mediavilla, 2010).

En este trabajo se indaga acerca de si existe una diferencia importante en los resultados educativos en función del entorno grupal del colegio, y si esta diferencia afecta a la significatividad de la titulación de la escuela, es decir, si afecta a la variable que indica que la escuela es de gestión privada o pública. Dado que se plantean distintas opciones de modelos, a continuación se describen las posibles variables que pueden llegar a utilizarse como "control" de tales opciones. Luego se presenta el detalle de las dos variables consideradas como principales en función del objetivo propuesto. En el cuadro 3 puede observarse una caracterización de la muestra en función de las diferentes variables explicativas.

a) Variables correspondientes al nivel alumnos (propias y vinculadas a características de sus hogares):

- Sexo (género): dicotómica. Vale 1 si el individuo es varón.

- Edad: continua. Edad del estudiante. Se calcula como la diferencia entre el año y mes de la prueba y el año y mes de nacimiento del estudiante, aplicando una fórmula especial, de modo de obtener variabilidad entre las observaciones ${ }^{8}$.

\footnotetext{
${ }^{8}$ Edad $=(100+$ año test - año nacimiento $)+($ mes test - mes nacimiento) $/ 12$.
}

- Nivel secundario superior (nivel_secundario): dicotómica. Vale 1 si el individuo se encuentra en el nivel "secundaria superior" (es decir, si está cursando alguno de los dos últimos años del nivel educativo medio) y 0 si se encuentra en el nivel "secundaria inferior" (es decir, si está cursando alguno de los tres primeros años del nivel educativo medio) ${ }^{9}$.

- Estatus ocupacional de los padres (HISEI): continua. Índice construido por la OCDE que representa dicho estatus. Surge de considerar el estatus más alto entre padre y madre.

- Nivel educativo de los padres (PARED): continua. Índice construido por la OCDE que representa dicho nivel medido como cantidad de años de estudio aprobados. Surge de considerar el nivel más alto entre padre y madre.

- Lugar de nacimiento (Nativo): dicotómica. Vale 1 si el individuo es nativo y 0 si es inmigrante de primera o segunda generación.

- Posesiones culturales del hogar (CUltPoss) ${ }^{10}$ : continua. Índice construido por la oCDE que representa la cantidad de tales posesiones (incluye si en el hogar hay disponibilidad de libros de literatura clásica y de poesía, y también si hay obras de arte). Un mayor valor del índice representa un mayor nivel cultural del hogar.

- Recursos educativos del hogar (HEDRES): continua. Índice construido por la OCDE que representa la cantidad de esos recursos (incluye si dispone de un escritorio y de un lugar tranquilo para estudiar, si cuenta con un computador para realizar tareas escolares, si este contiene un programa computacional educativo, y si posee libros de estudio y un

\footnotetext{
${ }^{9}$ Todos los alumnos evaluados tienen 15 años de edad, pero se dividen en tres grupos: aquellos que repitieron algún año escolar y asisten a cursos inferiores a los de su edad; aquellos que asisten al curso que les corresponde por edad y, según el año académico, se encuentran cursando el segundo año de secundaria, y aquellos que asisten al curso que les corresponde por edad, pero que según el año académico se encuentran cursando el tercer año de secundaria. Los dos primeros grupos mencionados asisten al nivel educativo secundario inferior (es decir, a alguno de los dos primeros años de nivel medio) y los que pertenecen al tercer grupo mencionado asisten al nivel educativo secundario superior (es decir, a alguno de los tres últimos años de nivel medio).

${ }^{10}$ Esta variable presenta algunos datos perdidos y, como el porcentaje de datos perdidos es bajo (3\% de las observaciones), se imputa el valor medio cuando el dato no se encuentra disponible. Asimismo, se añade una variable flag con el fin de incorporar la información de cuántas observaciones presentan datos imputados. Se la denomina CULTPOSS_o, es dicotómica y vale 1 si el valor de cuLTPOss es original y 0 en caso contrario.
} 
CUADRO 3

Caracterización de las observaciones en función de las diferentes variables explicativas

\begin{tabular}{|c|c|c|}
\hline Variables cuantitativas & Promedio & Desvío estándar \\
\hline & \multicolumn{2}{|c|}{ Variable dependiente } \\
\hline \multirow[t]{2}{*}{ Valores plausibles en ciencias } & 398,28 & 98,78 \\
\hline & \multicolumn{2}{|c|}{ Variables explicativas: nivel 1(alumnos) } \\
\hline Edad $($ Age $)$ & 15,69 & 0,28 \\
\hline Estatus ocupacional de los padres (HISEI) & 45,81 & 16,88 \\
\hline Nivel educativo de los padres (PARED) & 12,25 & 4,35 \\
\hline Posesiones culturales del hogar (CULTPOSs) & $-0,21$ & 0,87 \\
\hline Recursos educativos del hogar (HEDRES) & $-0,67$ & 1,05 \\
\hline Riqueza del hogar (WEALTH) & $-1,29$ & 0,89 \\
\hline Nivel socioeconómico del hogar (ESCS) & $-0,61$ & 1,14 \\
\hline \multirow[t]{2}{*}{ Posesiones del hogar: riqueza, recursos educativos y culturales (HOMEPOS) } & $-1,05$ & 1,01 \\
\hline & \multicolumn{2}{|c|}{ Variables independientes: nivel 2 (escuelas) } \\
\hline Tamaño de clase (CLSIZE) & 30,81 & 9,78 \\
\hline Proporción de alumnas & 0,53 & 0,16 \\
\hline (Proporción de niñas) & & \\
\hline Responsabilidad docente sobre el plan de estudio (RESPRES) & $-0,39$ & 0,51 \\
\hline Responsabilidad docente sobre los recursos (RESCURR) & 0,29 & 0,86 \\
\hline Calidad de los recursos educativos (SCMATEDU) & $-0,69$ & 1,34 \\
\hline Escasez de profesores (TCSHORT) & $-0,19$ & 0,97 \\
\hline Nivel socioeconómico promedio (PROESCS) & $-0,70$ & 0,78 \\
\hline \multirow[t]{2}{*}{ Variables cualitativas } & Categoría & Frecuencia \\
\hline & \multicolumn{2}{|c|}{ Variables explicativas: nivel 1 (alumnos) } \\
\hline Sexo & Varón & $46,2 \%$ \\
\hline (Género) & Mujer & $53,8 \%$ \\
\hline Nivel secundario superior & Superior & $71,2 \%$ \\
\hline (Nivel secundario) & Inferior & $28,8 \%$ \\
\hline \multirow{3}{*}{$\begin{array}{l}\text { Lugar de nacimiento } \\
\text { (Nativo) }\end{array}$} & Argentino & $97,5 \%$ \\
\hline & No argentino & $2,5 \%$ \\
\hline & \multicolumn{2}{|c|}{ Variables explicativas: nivel 2 (escuelas) } \\
\hline Tipo de gestión & Pública & $67,4 \%$ \\
\hline (Pública) & Privada & $32,6 \%$ \\
\hline
\end{tabular}

Fuente: elaboración propia sobre la base de datos del Programa para la Evaluación Internacional de Alumnos (PISA), 2006.

diccionario). Un mayor valor del índice representa un mayor nivel de recursos educativos en el hogar.

- Nivel de riqueza del hogar (WEALTH): continua. Índice construido por la oCDE que representa las posesiones existentes en la vivienda del estudiante (incluye si posee un cuarto propio, conexión a Internet, lavarropas, DVD, heladera con congelador, teléfono fijo y televisión por cable. Asimismo, considera cuántos teléfonos celulares, televisores, computadores y automóviles hay en el hogar). Un mayor valor del índice representa un mayor nivel económico. b) Variables correspondientes al nivel escuelas:

- Tamaño de la clase (CLSIZE) $)^{11}$ : indica el número promedio de alumnos por clase.

- Proporción de alumnas (proporción de niñas): indica la proporción de alumnas mujeres en la escuela.

\footnotetext{
${ }^{11}$ Esta variable y SCMATEDU presentan algunos (pocos) datos perdidos. Por ello, se imputa el valor de la media cuando la información no se halla disponible. Asimismo, se presentan dos variables flag (CLZISE_o y SCMATEDU_o) que, al igual que en el caso de cULTPOSS_o, informan si la observación presenta o no un dato imputado.
} 
- Responsabilidad docente respecto del plan de estudio (RESPRES): índice construido por la OCDE que representa el grado de responsabilidad del plantel docente en temas vinculados al plan de estudio. Mayores valores indican mayores niveles de responsabilidad.

- Responsabilidad docente en relación con los recurSOS (RESCURR): índice construido por la OCDE que representa el grado de responsabilidad del plantel docente en la asignación de los recursos. Mayores valores indican mayor responsabilidad.

- Calidad de los recursos educativos (scmatedu): índice construido por la oCDE que representa la cantidad y calidad de los recursos educativos de la escuela (incluye consideraciones sobre equipos de laboratorio, libros, computadoras, conexión a Internet, medios audiovisuales, entre otros). Valores positivos significan que la escuela posee condiciones favorables en cuanto a los recursos educativos, y valores negativos implican lo contrario. Asimismo, valores más positivos indican un mayor nivel de recursos educativos.

- Escasez de profesores (TCSHORT): índice construido por la oCDE que representa la escasez de profesores. Valores más positivos indican un mayor grado de problemática debido a la falta de profesores calificados.

Conviene aclarar que aquí no se incluyen variables referidas al rendimiento individual previo de los alumnos. Esto debido a que el rendimiento educativo suele ser recurrente, por lo que utilizar rendimiento individual pasado para explicar el actual puede resultar poco apropiado (Viego, 2006).

Respecto de las principales variables consideradas en este trabajo, en relación con la titularidad de los centros se cuenta con información sobre el tipo de gestión de cada escuela y esa información se representa por medio de una variable dicotómica llamada "pública", que toma el valor 1 si la escuela es de gestión pública y 0 si es de gestión privada. Por otra parte, en relación con el entorno escolar, se considera entorno a las características del alumnado a nivel de centro, ya que en la prueba PISA de 2006 no se reporta información referida a aulas. El entorno se representa mediante la variable "Nivel socioeconómico de la escuela". Para cuantificarlo se utiliza el promedio del indicador Escs en la escuela.

$\mathrm{El}$ indicador ESCS es construido por el equipo del programa PISA de la OCDE y resume la información de los índices HISEI, PARED y HOMEPOS. HISEI y PARED ya han sido explicados previamente. HOMEPOS resume la información de los índices WEALTH, HEDRES y CULTPOSS, y comprende también la cantidad de libros que hay en el hogar. En suma, ESCS representa el nivel socioeconómico del hogar. Este índice está confeccionado de manera tal que un valor positivo representa una situación en que el nivel socioeconómico del hogar sobrepasa el nivel promedio de los países de la OCDE, mientras que un valor negativo implica lo contrario. Asimismo, un mayor valor del índice representa un mayor nivel socioeconómico.

En los próximos párrafos se exponen los resultados de los modelos estimados. Como ya se ha mencionado, en el modelo 1 fue incorporada únicamente la constante. En los modelos siguientes se van incorporando paulatinamente variables explicativas. En principio, las referidas a los estudiantes y más tarde las vinculadas a las escuelas.

Por ello, a continuación se propone incluir como variables independientes a todas las variables correspondientes al nivel 1. En primer lugar, estas se incorporan con efectos fijos (modelo 2). En segundo lugar, se propone otro modelo (modelo 3$)^{12}$ en que se incluyen las mismas variables explicativas que en el modelo $2 \mathrm{y}$, al mismo tiempo, se incorporan efectos aleatorios en aquellas variables que resultaron ser estadísticamente significativas en el modelo 2. Finalmente, se analiza si dichos efectos aleatorios son también significativos. En el cuadro 4 se muestran los resultados de estos modelos.

En relación con el modelo 2, las variables que resultan significativas (con un nivel de confianza de 95\%) son "NivelSec", HISEI, PARED y HEDRES ${ }^{13}$. Entonces, a partir de los resultados encontrados y en términos resumidos, puede decirse que los individuos que habitan un hogar con mejor clima educativo (representado por el estatus ocupacional y el nivel educativo de sus padres) y mayores recursos educativos (representados por HEDRES) obtienen resultados académicos más elevados. Por ende y de acuerdo con el modelo 2, no existe equidad educativa, ya que los individuos obtienen resultados educativos diferentes en función de las características de sus hogares, y las escuelas no logran compensar estas diferencias de origen de manera que los resultados sean homogéneos. Asimismo, se observa que quienes ya están cursando el nivel educativo secundario superior también poseen un mejor desempeño.

\footnotetext{
12 Cabe resaltar que no existen problemas de multicolinealidad en los modelos 2 y 3 ; el resultado medio del factor de inflación de la varianza (FIV) es 1,2 y no supera el valor 5 para ninguna variable. El valor 5 de referencia se toma siguiendo a Calero y Escardíbul (2007). ${ }^{13} \mathrm{El}$ programa reporta el resultado de realizar una prueba de hipótesis individual para cada variable, en que se utiliza el estadístico $t$ y se plantea como hipótesis nula que el coeficiente es igual a 0.
} 
CUADRO 4

Coeficientes estimados en los modelos 2 al 5

(Estimación final de efectos fijos con errores estándar robustos a la heteroscedasticidad)

\begin{tabular}{|c|c|c|c|c|}
\hline \multirow{2}{*}{ Variables } & \multicolumn{4}{|c|}{ Coeficientes: parte fija } \\
\hline & Modelo 2 & Modelo 3 & Modelo 4 & Modelo 5 \\
\hline Constante & $\begin{array}{r}173,64 \\
(0,041)\end{array}$ & $\begin{array}{r}166,90 \\
(0,047)\end{array}$ & $\begin{array}{l}191,84 \\
(0,024)\end{array}$ & $\begin{array}{l}206,4 \\
(0,015)\end{array}$ \\
\hline Género & $\begin{array}{c}3,06 \\
(0,448)\end{array}$ & $\begin{array}{c}2,21 \\
(0,578)\end{array}$ & $\begin{array}{l}2,06 \\
(0,601)\end{array}$ & $\begin{array}{c}1,96 \\
(0,619)\end{array}$ \\
\hline Edad & $\begin{array}{l}4,91 \\
(0,330)\end{array}$ & $\begin{array}{c}5,39 \\
(0,281)\end{array}$ & $\begin{array}{l}5,65 \\
(0,260)\end{array}$ & $\begin{array}{c}5,32 \\
(0,293)\end{array}$ \\
\hline NivelSec & $\begin{array}{l}67,65 \\
(0,000)\end{array}$ & $\begin{array}{l}67,64 \\
(0,000)\end{array}$ & $\begin{array}{l}65,02 \\
(0,000)\end{array}$ & $\begin{array}{l}63,56 \\
(0,000)\end{array}$ \\
\hline HISEI & $\begin{array}{c}0,35 \\
(0,003)\end{array}$ & $\begin{array}{c}0,32 \\
(0,009)\end{array}$ & $\begin{array}{c}0,33 \\
(0,006)\end{array}$ & $\begin{array}{c}0,29 \\
(0,016)\end{array}$ \\
\hline PARED & $\begin{array}{c}1,92 \\
(0,000)\end{array}$ & $\begin{array}{l}2,02 \\
(0,000)\end{array}$ & $\begin{array}{l}1,91 \\
(0,000)\end{array}$ & $\begin{array}{l}1,81 \\
(0,000)\end{array}$ \\
\hline NATIVO & $\begin{array}{l}-3,58 \\
(0,652)\end{array}$ & $\begin{array}{l}-3,07 \\
(0,701)\end{array}$ & $\begin{array}{l}-2,42 \\
(0,764)\end{array}$ & $\begin{array}{l}-1,67 \\
(0,837)\end{array}$ \\
\hline CULPOSS & $\begin{array}{c}2,64 \\
(0,221)\end{array}$ & $\begin{array}{c}2,56 \\
(0,252)\end{array}$ & $\begin{array}{l}2,42 \\
(0,276)\end{array}$ & $\begin{array}{l}2,09 \\
(0,348)\end{array}$ \\
\hline HEDRES & $\begin{array}{c}7,64 \\
(0,001)\end{array}$ & $\begin{array}{c}7,74 \\
(0,000)\end{array}$ & $\begin{array}{c}7,73 \\
(0,000)\end{array}$ & $\begin{array}{c}7,86 \\
(0,000)\end{array}$ \\
\hline WEALTH & $\begin{array}{l}-1,86 \\
(0,375)\end{array}$ & $\begin{array}{l}-1,50 \\
(0,462)\end{array}$ & $\begin{array}{l}-1,84 \\
(0,364)\end{array}$ & $\begin{array}{l}-2,35 \\
(0,244)\end{array}$ \\
\hline CULTPOSS_O & $\begin{array}{l}51,56 \\
(0,000)\end{array}$ & $\begin{array}{l}51,16 \\
(0,000)\end{array}$ & $\begin{array}{l}50,24 \\
(0,000)\end{array}$ & $\begin{array}{l}50,02 \\
(0,000)\end{array}$ \\
\hline CLSIZE & & & $\begin{array}{c}1,37 \\
(0,014)\end{array}$ & $\begin{array}{c}1,28 \\
(0,009)\end{array}$ \\
\hline RESCURR & & & $\begin{array}{c}8,39 \\
(0,120)\end{array}$ & $\begin{array}{l}7,0 \\
(0,192)\end{array}$ \\
\hline SCMATEDU & & & $\begin{array}{c}8,09 \\
(0,019)\end{array}$ & $\begin{array}{l}7,15 \\
(0,030)\end{array}$ \\
\hline PÚBLICA & & & $\begin{array}{r}-38,39 \\
(0,001)\end{array}$ & $\begin{array}{r}-15,07 \\
(0,138)\end{array}$ \\
\hline CLSIZE_O & & & $\begin{array}{r}-18,87 \\
(0,099)\end{array}$ & $\begin{array}{r}-27,97 \\
(0,055)\end{array}$ \\
\hline SCMATEDU_O & & & $\begin{array}{r}-17,49 \\
(0,383)\end{array}$ & $\begin{array}{r}-11,02 \\
(0,602)\end{array}$ \\
\hline PROESCS & & & & $\begin{array}{l}25,90 \\
(0,001)\end{array}$ \\
\hline
\end{tabular}

Fuente: elaboración propia sobre la base de datos del Programa para la Evaluación Internacional de Alumnos (PISA), 2006.

Nota: valor $p$ entre paréntesis.

Respecto del modelo 3 puede decirse que los efectos aleatorios incluidos en él son estadísticamente significativos a menos del $5 \%{ }^{14}$. Esto implica que el grado en

\footnotetext{
${ }^{14} \mathrm{El}$ programa WHLM reporta un cuadro en que se presenta el resultado de una prueba del chi cuadrado para analizar el efecto aleatorio de cada variable. En esa prueba se testean, para cada variable, los coeficientes de todos los centros a la vez; la hipótesis nula es que los coeficientes de todas las escuelas son iguales, es decir, que la "parte aleatoria" del coeficiente en cada centro es 0 (la distancia entre el coeficiente de cada centro y el coeficiente promedio es nula). Entonces, caer en la zona de rechazo (como ocurre en el caso de las tres variables consideradas) implica que esta hipótesis no es aceptada y resulta
}

que la escasez de recursos educativos en el hogar o su entorno desfavorable para estudiar pueden disminuir el rendimiento de los alumnos es distinto en función de la escuela a la que estos asisten. Es decir, quienes poseen limitaciones de origen pueden ser compensados en mayor o menor medida en su escuela, o directamente no ser compensados de forma alguna.

En tercer lugar, y a fin de mejorar la explicación brindada por el modelo anterior, se lo amplía mediante la inclusión de variables referidas al nivel de centros (modelo $4^{15}$ ), pero aún no se incluye una variable con que se procure cuantificar y medir el "entorno".

Como se puede observar en el cuadro 4, se incorporan las variables CLSIZE, RESCURR, SCMATEDU y "Pública" ${ }^{16}$. Todas ellas resultan ser significativas al 5\%, con excepción de RESCURR. Por otra parte, se observa que la significatividad y el efecto de las variables correspondientes al nivel 1 no se han visto alterados. Calero y Escardíbul (2007) explican que este es un resultado esperado en los modelos multinivel.

La información extra que brinda el modelo 4 en relación con el modelo 3 es que el rendimiento de los individuos es mayor en las escuelas que: i) albergan en cada aula una mayor cantidad de alumnos; ii) poseen una mejor calidad de recursos educacionales, y iii) son de gestión privada.

Es destacable el dato de que los alumnos que asisten a escuelas de gestión pública, ceteris paribus, obtienen 38 puntos menos que los que concurren a escuelas de gestión privada. Sin embargo, es prudente no atribuirle una importancia muy elevada al efecto del tipo de gestión escolar en el desempeño académico, sin antes analizar qué ocurre con esta variable al incluir en el modelo 4 información sobre el "entorno", ya que — como se ha mencionado previamente - hay evidencia empírica de que la incorporación del "efecto de pares" resta o elimina la significatividad de la titulación de la escuela.

A continuación, con el fin de captar si existen efectos vinculados al entorno escolar, en el modelo 4 se incorpora una variable con la que se procura cuantificar

legítimo enunciar que existen diferencias en las pendientes de las regresiones de las escuelas.

15 No existen problemas de multicolinealidad; el valor medio del factor de inflación de la varianza (FIV) es 1,21 y no supera el valor 5 para ninguna variable.

${ }^{16}$ No se incorpora la variable "proporción de niñas" porque, según los modelos 2 y 3 , el género no es un factor significativo. Asimismo, no se incluye la variable RESPRES por estar altamente correlacionada con RESCURR (coeficiente de Pearson positivo y significativo al 1\%) y no se incluye TCSHORT por estar altamente correlacionada con SCMATEDU (coeficiente de Pearson negativo y significativo al 1\%). 
el "efecto de pares". Esa variable es "Promedio de escs" y ella misma resulta ser significativa (valor $\mathrm{P}=0,001$ ). Este nuevo modelo es denominado modelo $5^{17}$.

Entonces, se encuentra evidencia en favor del efecto de pares. Asimismo, la inclusión de esta variable provoca la disminución de la significatividad de la variable "Pública", de modo que el valor P de "Pública" pasa de 0,001 (modelo 4) a 0,138, dejando de ser "Pública" una variable significativa. Este resultado da la pauta de que no es tan importante la titularidad de la escuela en sí misma, sino las características de las familias de los alumnos que asisten a cada tipo de escuela.

Finalmente, se comparan los modelos propuestos con el fin de elegir el más apropiado para elaborar las conclusiones finales. En el cuadro 5 puede observarse que el modelo 5 resulta ser más adecuado debido a los siguientes motivos:

i) Globalmente, el modelo 5 es el más explicativo de los tres (36\% sobre el modelo nulo). Es decir, que deja sin explicar un menor porcentaje de varianza residual en relación con el modelo nulo.

ii) De acuerdo con el objetivo de investigación, interesa especialmente el efecto del nivel escuelas en el rendimiento; por ello el modelo 5 resulta también

${ }^{17}$ No existen problemas de multicolinealidad, el FIV promedio es igual a 1,27 y no supera el valor 5 para ninguna variable. ser más adecuado, ya que brinda una mayor cantidad de información al respecto. Por una parte, las variables utilizadas pertenecientes al nivel de escuelas (nivel 2) explican un alto porcentaje de la variabilidad de los resultados de las pruebas entre las escuelas (52\%) y ese porcentaje supera al correspondiente en los modelos restantes. Por otra parte, si se observa la composición del porcentaje de varianza residual no explicada en cada caso, el menor peso del nivel 2 corresponde al modelo 5 $(28 \%)$.

Por lo tanto, el modelo 5 es el utilizado para analizar el papel de las variables definidas como principales en este trabajo. En el cuadro 5 se pueden observar los coeficientes estimados mediante dicho modelo.

En función de los resultados que ofrece el modelo escogido (modelo 5), puede decirse que la titularidad de la escuela no es un determinante significativo del rendimiento escolar. Mientras que el entorno socioeconómico al que se enfrentan los alumnos de cada escuela sí resulta ser una variable explicativa importante de su desempeño académico y representa el denominado efecto de pares. Por lo tanto, se presenta una respuesta en favor de la hipótesis de que el factor determinante de los resultados de calidad en el rendimiento no es el tipo de gestión de la escuela en sí mismo, sino el tipo de alumnos que asiste a cada clase de escuela (de gestión pública o privada).

CUADRO 5

Principales resultados de los modelos no nulos

\begin{tabular}{lcccc}
\hline & Modelo 2 & Modelo 3 & Modelo 4 & Modelo 5 \\
\hline Varianza residual (nivel 1) & 3621,43 & 3500,41 & 3520,14 & 3517,26 \\
Varianza residual (nivel 2) & 2922,06 & 4242,93 & 3307,76 & 2795,64 \\
Varianza residual total & 6543,49 & 7743,34 & 6827,90 & 6312,90 \\
Porcentaje de varianza residual explicado por las variables sobre & 34 & 22 & 31 & 36 \\
el modelo nulo: disminución en la varianza residual total & & &
\end{tabular}

Comparación con el modelo nulo

\begin{tabular}{|c|c|c|c|c|}
\hline $\begin{array}{l}\text { Porcentaje de varianza residual explicado por las variables sobre } \\
\text { el modelo nulo: nivel } 1 \text { (alumnos) }\end{array}$ & 12 & 15 & 15 & 15 \\
\hline $\begin{array}{l}\text { Porcentaje de varianza residual explicado por las variables sobre } \\
\text { el modelo nulo: nivel } 2 \text { (escuelas) }\end{array}$ & 50 & 27 & 43 & 52 \\
\hline Porcentaje de varianza residual total no explicado por el modelo & 66 & 78 & 69 & 64 \\
\hline
\end{tabular}

Composición del porcentaje de varianza residual total no explicado por el modelo

\begin{tabular}{lccc}
\hline $\begin{array}{l}\text { Porcentaje de varianza residual existente por diferencias entre } \\
\text { alumnos (dentro de las escuelas) } \\
\text { Porcentaje de varianza residual existente por diferencias entre escuelas }\end{array}$ & 36 & 35 & 35 \\
\hline
\end{tabular}

Fuente: elaboración propia sobre la base de datos del Programa para la Evaluación Internacional de Alumnos (PISA), 2006. 


\section{VI}

\section{Conclusiones}

A lo largo de este trabajo se han estudiado los determinantes del rendimiento escolar en el nivel medio de la Argentina, utilizando datos de la prueba PISA de 2006 y con el fin de responder a esta pregunta: ¿Es significativo el tipo de gestión escolar a la hora de explicar los resultados educativos?

La evidencia aquí presentada favorece la hipótesis planteada en la Introducción y da cuenta de que la correlación entre la clase de gestión de la escuela y el rendimiento educativo se disipa al considerar el entorno socioeconómico escolar. Esto parece indicar que las escuelas de gestión privada brindan una educación de mejor calidad que las escuelas de gestión pública, pero no debido a su forma de organización, sino a que albergan alumnos con mayores ventajas, es decir, con mejores condiciones de origen para desarrollarse como estudiantes y alcanzar un desempeño alto.

De aquí surge una cuestión interesante para ser analizada en futuras investigaciones: si bien el tipo de organización escolar de gestión privada no parece ser un determinante decisivo del rendimiento, sí es verdad que ofrece algún tipo de señal por la que luego se agrupan allí individuos de cierto estatus socioeconómico. Es decir, en el mecanismo por el que las familias eligen este tipo de escuelas y, muchas veces, las escuelas eligen a sus alumnos, puede estar la explicación del entorno favorable que se crea para estudiar.

Asimismo, de acuerdo con las consideraciones aquí expuestas, puede decirse que, por una parte, es necesario que se implementen políticas que exceden el ámbito educativo, porque el nivel socioeconómico se presenta como un determinante muy decisivo del rendimiento educativo. Sin embargo, entre los resultados se ha destacado el hecho de que las escuelas difieren en su capacidad para compensar las desigualdades de origen. Por ello puede afirmarse que existe margen para la política educativa. Es decir, dado que algunas escuelas tienen un mejor desempeño que otras, puede afirmarse que hay espacio para ir en procura de mejoras que, al menos, equiparen la actuación entre las escuelas.

Respecto de cuáles políticas educativas llevar a cabo, de acuerdo con la información presentada en este trabajo puede vislumbrarse que un aspecto a tener en cuenta es la calidad de los recursos educativos que posee cada escuela. Sin embargo, es necesario un estudio más detallado para precisar qué aspectos deberían priorizarse. Será trabajo de futuras investigaciones incorporar más variables en el nivel 2 de análisis (escuelas), a objeto de poder plantear con mayor exactitud consideraciones de política.

Finalmente, resta mencionar que muchos gobiernos pueden estar interesados en lograr un nivel mínimo en la educación de los alumnos. Para evaluar el grado en que se cumple esa meta y las condiciones determinantes del éxito o fracaso escolar en ese sentido, pueden estudiarse los factores que influyen específicamente en la probabilidad de que un individuo fracase en su carrera escolar ${ }^{18}$. Esto también será fuente de futuros trabajos.

\footnotetext{
${ }^{18}$ Esto es posible porque se han desarrollado programas computacionales que permiten utilizar modelos de regresión logística en el análisis multinivel.
}

Abdul-Hamid, H. (2007), "Evaluación de lo preparada que está Argentina para la economía del conocimiento: medición del conocimiento y las habilidades de los alumnos en matemáticas y ciencias con resultados de PISA 2000", Bienestar y política social, vol. 3, № 2, México, D.F., Conferencia Interamericana de Seguridad Social/Universidad Iberoamericana.

Calero, J., A. Choi y S. Waisgrais (2010), “¿Qué determina el fracaso escolar en España? Un estudio a través de PISA-2006” [en línea] http://www.um.es/dp-hacienda/eep2010/comunicaciones/ eep2010-6.pdf.

(2009) "Determinantes del rendimiento educativo del alumnado de origen nacional e inmigrante en PISA-2006", Cuadernos económicos, $\mathrm{N}^{\circ}$ 77, Madrid, Información Comercial Española, Ministerio de Industria, Turismo y Comercio.

Calero, J. y J.O. Escardíbul (2007), "Evaluación de servicios educativos: el rendimiento en los centros públicos y privados medido en PISA-2003", Hacienda pública española, vol. 183, № 4, Madrid, Instituto de Estudios Fiscales.

Cervini, R. (2010), "El 'efecto escuela' en la educación primaria y secundaria: el caso de Argentina", Revista electrónica iberoamericana sobre calidad, eficiencia y cambio en educación (REICE), vol. 8, $\mathrm{N}^{\mathrm{o}}$ 1, Madrid, Red Iberoamericana de Investigación sobre Cambio y Eficacia Escolar.

(2009), "Comparando la inequidad en los logros escolares de la educación primaria y secundaria en Argentina: un estudio 
multinivel", Revista electrónica iberoamericana sobre calidad, eficiencia y cambio en la educación (REICE), vol. 7, $\mathrm{N}^{\mathrm{o}} 1$, Madrid, Red Iberoamericana de Investigación sobre Cambio y Eficacia Escolar.

(2006), "Los efectos de la escuela y del aula sobre el logro en matemáticas y en lengua de la educación secundaria. Un modelo multinivel", Perfiles educativos, vol. 28, № 112, México, D.F., Universidad Nacional Autónoma de México.

(2005a), "Nivel y variación de la equidad en la educación media de Argentina", Revista iberoamericana de educación, Madrid, Organización de Estados Iberoamericanos (OEI).

(2005b), "Variación de la equidad en resultados cognitivos y no cognitivos de la educación media de Argentina", Revista electrónica de investigación educativa, vol. 7, No 1 [en línea] http://redie.uabc.mx/vol7no1/contenido-cervini3.html.

(2002a), "Desigualdades socioculturales en el aprendizaje de matemática y lengua de la educación secundaria en Argentina: un modelo de tres niveles", Revista electrónica de investigación y evaluación educativa (RELIEVE), vol. 8, No 2, Valencia, Universidad de Valencia.

(2002b), "Desigualdades en el logro académico y reproducción cultural en Argentina. Un modelo de tres niveles", Revista mexicana de investigación educativa, vol. 7, $\mathrm{N}^{\circ}$ 16, México, D.F., Consejo Mexicano de Investigación Educativa.

(1999), Calidad y equidad en la educación básica en la Argentina, Buenos Aires, Ministerio de Cultura y Educación de la Nación.

De la Cruz, F. (2008), "Modelos multinivel” [en línea] http://rpe. epiredperu.net/rpe_ediciones/v12_n03_2008/AR1.pdf

Diez Roux, A. (2002), "A glossary for multilevel analysis", Journal of Epidemiology and Community Health, vol. 56, $\mathrm{N}^{\circ}$ 8, BMJ Publishing Group.

Fernández Aguerre, T. (2002), "Determinantes sociales e institucionales de la desigualdad educativa en sexto año de educación primaria de Argentina y Uruguay, 1999. Una aproximación mediante un modelo de regresión logística", Revista mexicana de investigación educativa, vol. 7, $\mathrm{N}^{\circ}$ 16, México, D.F., Consejo Mexicano de Investigación Educativa.

Formichella, M. (2010), "Educación y desarrollo: análisis desde la perspectiva de la equidad educativa interna y del mercado laboral", tesis, Buenos Aires, Universidad Nacional del Sur.

Gertel, H. y otros (2007a), "El rendimiento escolar de la población de estudiantes de la educación básica en Argentina: ¿cómo contribuye la gestión de la escuela?", IX Jornadas Argentinas de Estudios de Población, Córdoba, Asociación Argentina de Estudios de Población.

(2007b), "Incidencia de la gestión sobre el rendimiento escolar en la escuela argentina. El mensaje de las pruebas internacionales y nacionales" [en línea] http://www.aaep.org.ar.

(2007c), "Los factores determinantes del rendimiento escolar al término de la educación básica en Argentina. Una aplicación de técnicas de análisis jerárquico de datos", Anales de las XVI Jornadas de la Asociación de Economía de la Educación.

(2006), "Análisis multinivel del rendimiento escolar al término de la educación básica en Argentina", documento presentado en la Reunión anual de la Asociación Argentina de Economía Política [en línea] en http://www.aaep.org.ar/espa/ anales/works06/Gertel_Giuliodori_Herrero.pdf

Hox, J.J (1995), Applied Multilevel Analysis, Amsterdam, TT-Publikaties.

Martínez Arias, R. (2006), "La metodología de los estudios PISA", Revista de educación, volumen extraordinario, Madrid, Universidad Complutense de Madrid.

Mediavilla, M. (2010), "Las becas y ayudas al estudio como elemento determinante de la continuidad escolar en el nivel secundario post-obligatorio. Un análisis de sensibilidad a partir de la aplicación del Propensity Score Matching", Investigaciones de economía de la educación, Asociación de Economía de la Educación.

oCDE (Organización de Cooperación y Desarrollo Económicos) (2006a), El programa PISA de la OCDE. ¿Qué es y para qué sirve?, París. (2006b), Informe PISA 2006: competencias científicas para el mundo de mañana, París.

(2006c), PISA Data Análisis Manual. sPSs, Second Edition, París. (2003), PISA 2003. Manual de análisis de datos, París.

Santos, M. (2007), "Quality of education in Argentina: determinants and distribution using PISA 2000 test scores", Well-being and Social Policy, vol. 3, No 1, México, D.F., Conferencia Interamericana de Seguridad Social/Universidad Iberoamericana.

Viego, V. (2006), "Comentario al trabajo 'Análisis multinivel del rendimiento escolar al término de la educación básica en Argentina"” [en línea] www.aaep.org.ar.

Wößmann, L. y T. Fuchs (2005), "Families, schools, and primaryschool learning: evidence for Argentina and Colombia in an international perspective", Policy Research Working Paper Series, $\mathrm{N}^{\circ} 3537$, Washington, D.C., Banco Mundial. 